\title{
Long-term efficacy of psychological treatments for binge eating disorder
}

Anja Hilbert, Monica E. Bishop, Richard I. Stein, Marian Tanofsky-Kraff, Anne K. Swenson,

R. Robinson Welch and Denise E. Wilfley

\section{Background}

The long-term efficacy of psychological treatments for binge eating disorder remains largely unknown.

\section{Aims \\ To examine the long-term efficacy of out-patient group cognitive-behavioural therapy (CBT) and group interpersonal psychotherapy (IPT) for binge eating disorder and to analyse predictors of long-term non-response. \\ Method \\ Ninety people with binge eating disorder were assessed 4 years after treatment cessation within a randomised trial (trial registration: NCT01208272).}

\section{Results}

Participants showed substantial long-term recovery, partial remission, clinically significant improvement and significant reductions in associated psychopathology, despite relapse tendencies in single secondary outcomes. Body mass index remained stable. While the IPT group demonstrated an improvement in eating disorder symptoms over the follow-up period, the CBT group reported a worsening of symptoms, but treatments did not differ at any time point.

\section{Conclusions}

The results document the long-term efficacy of out-patient CBT and IPT for binge eating disorder. Further research is warranted to elucidate the time course and mechanisms of change of these treatments for binge eating disorder.

\section{Declaration of interest}

\section{None.}

Binge eating disorder, defined in the DSM-IV as a provisional diagnosis in need of further study, is characterised by recurrent binge eating that occurs in the absence of regular compensatory behaviours. ${ }^{1}$ Ample evidence indicates that binge eating disorder is a clinically significant disorder, associated with eating disorder and general psychopathology, psychiatric comorbidity, overweight and obesity and impaired quality of life. ${ }^{2,3}$ According to current reviews, ${ }^{4,5}$ meta-analyses ${ }^{6,7}$ and clinical treatment guidelines, ${ }^{8}$ cognitive-behavioural therapy (CBT) is considered the firstline specialty treatment for binge eating disorder. Cognitivebehavioural therapy produces substantial improvements in binge eating, associated psychopathology and psychosocial functioning, and modest weight loss has been documented in those who achieve abstinence from binge eating. ${ }^{9,10}$ Virtually identical gains have been found for interpersonal psychotherapy (IPT), a theoretically and procedurally distinct therapeutic approach. ${ }^{9,10}$ For both CBT and IPT, stability of treatment effects has been documented in randomised controlled trials (RCTs) over a period of up to 2 years following treatment cessation, ${ }^{10,11}$ yet longer-term effects remain unknown. The current study sought to examine the long-term effects of out-patient group CBT and IPT in a randomised controlled binge eating disorder psychotherapy trial (trial registration: NCT01208272). ${ }^{9}$

\section{Method}

\section{Participants and procedure}

Participants were the first 90 patients (55.6\%; the first five cohorts) out of a larger $(n=162)$ treatment trial for overweight people with binge eating disorder, recruited in New Haven, Connecticut, USA (for methodological detail see the main report ${ }^{9}$ ). As this long-term follow-up study was not part of the initial two-site clinical trial, funded by the National Institutes of Health (NIH), participants were re-consented with a separate informed consent approved by the San Diego State University
Institutional Review Board. Owing to the unfunded nature of this follow-up study, we opted to only enrol patients from the New Haven site. All participants met DSM-IV-TR diagnostic criteria for binge eating disorder ${ }^{1}$ and were, after stratification by gender, randomised to either CBT or IPT ( $n=45$ each). Both treatments were manual-based and consisted of 20 weekly 90-min group sessions and three additional individual sessions.

For the present study, patients were contacted for a long-term follow-up assessment approximately 4 years after treatment cessation (i.e. a mean of 46.0 months). As illustrated in Fig. 1, of the 90 patients included in this study, $77(85.6 \%)$ could be contacted (i.e. no current contact information: 10; incapacitated or deceased: 3). Of the 77 individuals contacted, 58 (75.3\%) completed the long-term follow-up assessment. Completer rates for post-treatment assessment were $89 / 90(98.9 \%)$ and for the 1-year follow-up assessment 77/90 (85.6\%). Eighty-three $(92.2 \%)$ of the 90 patients had completed treatment. Treatment and assessment completion rates did not differ by group (all $P>0.05)$.

\section{Assessments and procedures}

Outcome analyses included pre-treatment, post-treatment, 1-year follow-up and long-term follow-up assessments. The long-term follow-up assessment included a telephone interview and selfreport questionnaires, whereas all other assessments involved inperson diagnostic visits and self-report questionnaires. All structured clinical interviews were conducted by trained assessors (Bachelor level or higher) who received ongoing supervision to ensure standardised administration.

\section{Eating disorder psychopathology}

The diagnostic version of the Eating Disorder Examination (EDE 12.0D), ${ }^{12}$ a semi-structured interview with good reliability and validity, was used to assess days with objective bulimic episodes (i.e. eating an unusually large amount of food, accompanied by 


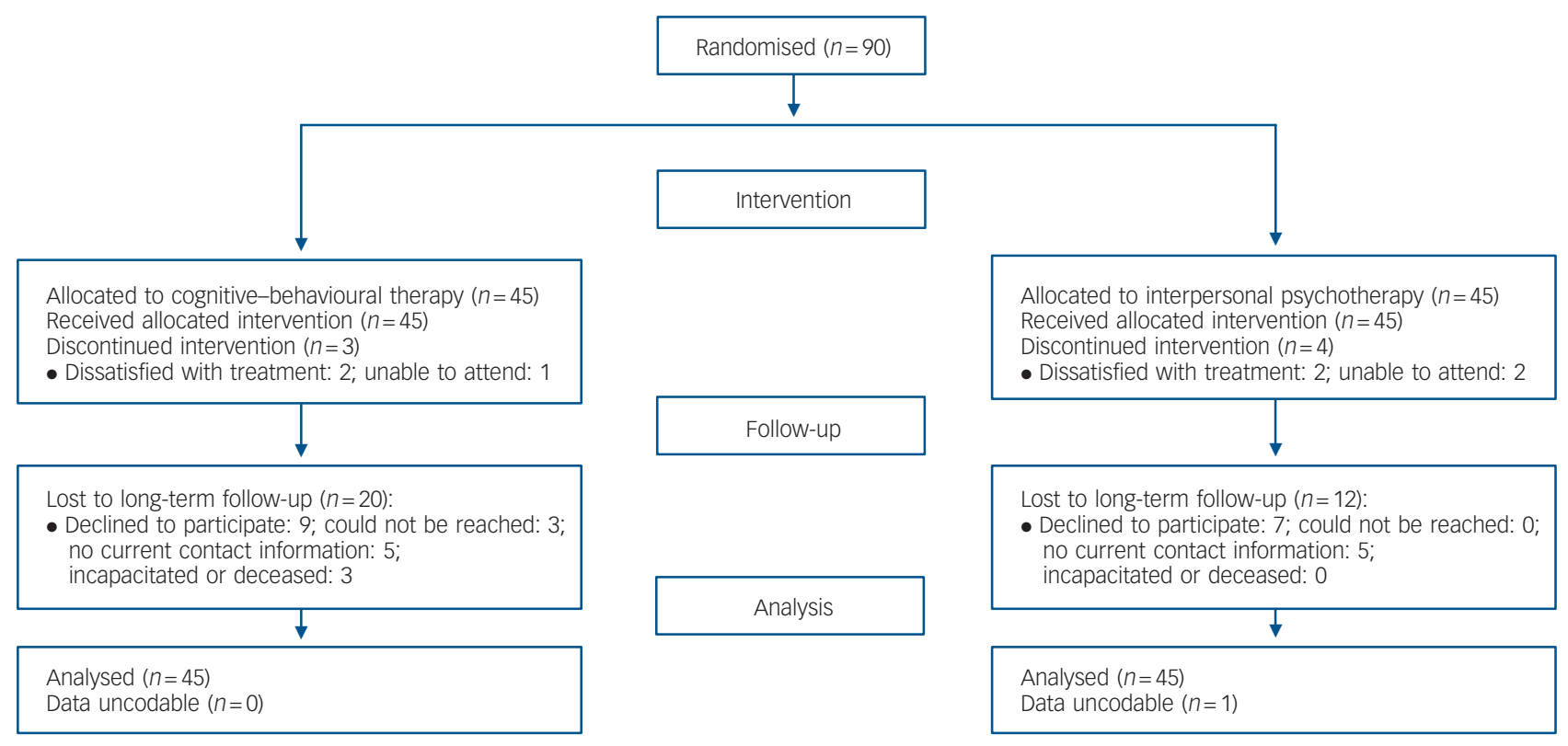

\section{Fig. 1 CONSORT flow diagram.}

Participation rates for the full study sample at pre-treatment, post-treatment, and 1-year follow-up assessment are described in the main study. ${ }^{9}$

a sense of loss of control over eating). ${ }^{1}$ The primary outcome variables of recovered and remitted were based on the EDE assessment of days with objective bulimic episodes over the previous 28 days (see Data analysis). Secondary outcome variables included the number of days with objective bulimic episodes over the previous 28 days and a composite shape/weight concern score, both derived from the EDE. For descriptive purposes, DSM-IV-TR diagnoses of binge eating disorder, bulimia nervosa, anorexia nervosa and eating disorder not otherwise specified were determined with the abbreviated EDE. Objective bulimic episode days and episodes were comprehensively assessed over the previous 6 months, whereas purging behaviour (i.e. self-induced vomiting, laxative or diuretic misuse), fasting, intense exercising, importance of shape or weight, fear of weight gain, feelings of fatness, maintained low weight, and menstruation were assessed over the previous 28 days and, if positive, over the previous 3 months.

In addition, the self-report version of the EDE, the Eating Disorder Examination Questionnaire (EDE-Q), ${ }^{13}$ was used to assess the associated eating disorder psychopathology on the four subscales of restraint, eating concern, shape concern and weight concern, and on global eating disorder psychopathology (an average of the four subscales). The EDE-Q indicators have demonstrated adequate internal consistency, convergent validity and sensitivity to change. ${ }^{14}$ The EDE weight/shape composite and the EDE-Q indicators were based on the previous 28 days and ranged from 0 to 6 , with higher scores indicating greater psychopathology.

\section{General psychopathology}

The depression and anxiety subscales of the Brief Symptom Inventory $(\mathrm{BSI})^{15}$ were used for assessment of general psychopathology. Subscale scores were used as secondary outcomes; they ranged from 0 to 4 , with higher scores indicating more severe psychiatric symptoms. Both subscales have adequate internal consistency, convergent validity and sensitivity to change. ${ }^{16}$

\section{Body mass index}

Body mass index (BMI, $\mathrm{kg} / \mathrm{m}^{2}$ ), a proxy for body fat, was used as a secondary outcome. Body mass index was calculated from weight (self-reported at long-term follow-up and measured at all other time points) and height (measured at pre-treatment). For diagnosis of anorexia nervosa (see above), underweight was determined as $\mathrm{BMI}<18.5 \mathrm{~kg} / \mathrm{m}^{2}$. Prior to treatment, measured and self-reported body weight were highly associated $(r=0.99$, $P<0.001)$.

\section{Healthcare utilisation}

At long-term follow-up, healthcare utilisation for eating or weight problems was retrospectively assessed for the time after 1-year follow-up. Use of psychotherapy, pharmacotherapy, consultation (for example with a dietician) and/or alternative treatment (for example hypnosis) and any of these treatments was determined in a dichotomous format $(0$, no; 1 , yes).

\section{Data analysis}

Preliminary analyses served (a) to compare patients randomised to CBT $v$. IPT ( $n=45$ each) on sociodemographic characteristics (age, gender), drop-out from treatment, adherence (i.e. number of sessions attended), and pre-treatment level of any outcome variable (univariate general linear model (GLM) analyses or $\chi^{2}$ analyses by treatment (CBT, IPT)); (b) to compare participants who were included in the long-term follow-up assessment $v$. those who were not included ( $n=90 v$. 72) by treatment on the same variables and outcome variables at all time points (univariate GLM or generalised linear model analyses of sample (included, not-included) $\times$ treatment $(\mathrm{CBT}$, IPT) for continuous or binary variables, respectively); and (c) to compare participants with completed $v$. non-completed long-term follow-up assessment ( $n=58 v .32)$ by treatment on these same variables and outcome variables at pre-treatment, post-treatment, and 1-year follow-up (univariate GLM or generalised linear model analyses of assessment (completed, not-completed) $\times$ treatment $($ CBT, IPT) for continuous or binary variables, respectively).

Statistical analyses were based on the generalised estimating equations (GEE) approach for the primary (categorical) outcome variables and on hierarchical linear modelling (HLM) for the 
secondary (continuous) outcome variables. Both intention-totreat approaches allow data from participants with missing data at some, but not all, time points to remain in the analyses. In addition, both approaches correct for the dependency of observations within participants, in GEE analysis by assuming an exchangeable working correlation structure, and in HLM by allowing the regression coefficients to vary between participants. For the efficacy analyses, the assessment completer sample size at long-term follow-up provided $80 \%$ power to detect a medium-to-large effect size of treatment difference $(d=0.76$; $n=25$ for CBT, $n=33$ for IPT).

The primary categorical outcome variables were analysed using GEE logistic regression models (i.e. logit link function and binomial error distribution) that included treatment (CBT, IPT) $\times$ time (post-treatment, 1-year follow-up, long-term followup) and the respective main effects as predictors. Least significant difference tests were used for post hoc analyses in case of significant higher-order effects. Consistent with the main outcome report, ${ }^{9}$ the primary outcome included three variables, all determined at post-treatment, 1-year follow-up and long-term follow-up: recovered (i.e. no objective bulimic episodes in the previous month), improved to subclinical binge eating (i.e. $<4$ objective bulimic episode days in the previous month) and being at or below a comparative level of eating disorder attitudes and behaviours. The latter rating was made based on whether the global EDE-Q score was at or below the global EDE-Q score of overweight treatment-seeking individuals without binge eating disorder who had a similar sociodemographic profile as the patients in the current study (global EDE-Q score, 2.47). ${ }^{17}$

The secondary continuous outcome variables were analysed using HLM of treatment (CBT, IPT) $\times$ time (pre-treatment, post-treatment, 1-year follow-up, long-term follow-up), with patients nested within time. In this analysis, time and treatment were treated as fixed factors and patients as a random factor. Least significant difference tests were used for post hoc analyses.

To ensure that the results from the primary and secondary intention-to-treat analyses were robust, three sensitivity analyses ${ }^{18}$ were conducted in addition, handling missing data as follows: (a) with missing data multiply imputed, creating five completed data-sets via an iterative Markov Chain Monte Carlo method; (b) with last observations carried forward; and (c) with no replacement values for missing data (i.e. completer analysis only including participants who had completed all assessments). Outcome analyses were performed on all sensitivity data-sets for inspection of significance. Results were reported only if different from those of the primary and secondary intention-to-treat analyses described above. Completer effect sizes were computed as Pearson's $r$ (small, $r \geqslant 0.10$; medium, $\geqslant 0.30$; large, $\geqslant 0.50$ ) or Cohen's $d$ (small, $d \geqslant 0.20$; medium, $\geqslant 0.50$; large, $\geqslant 0.80$ ). ${ }^{19}$
All analyses were performed with PASW 18.0 for Windows. A two-tailed significance level of $\alpha<0.05$ was applied to all statistical tests. In order to avoid $\alpha$ inflation by multiple testing, the significance level was adjusted to a two-tailed $\alpha<0.01$ for post hoc tests.

\section{Results}

\section{Randomisation, attrition and sampling}

Patients randomised to CBT $v$. IPT did not differ with regard to sociodemographic characteristics (Table 1) or any pre-treatment primary or secondary outcome variable (all $P>0.05$ ). There were no significant differences in drop-out (CBT: 3 (6.7\%); IPT: 4 $(8.9 \%))$ or adherence to treatment (CBT: 16.8 (s.d.=3.0); IPT: 17.7 (s.d. $=3.9$ ) sessions; all $P>0.05$ ).

The 90 patients who were included in the long-term follow-up assessment did not significantly differ from those who were not included in the follow-up assessment $(n=72)$ on pre-treatment characteristics, drop-out and adherence, and no interaction effects with treatment condition were observed (sample (included, notincluded $) \times$ treatment $(\mathrm{CBT}$, IPT); all $P>0.05)$. The 58 patients who completed the long-term follow-up assessment did not significantly differ from the 32 assessment non-completers on sociodemographic characteristics, drop-out, adherence, missing data, or primary and secondary outcomes at pre-treatment, post-treatment, or 1-year follow-up, and no interaction effects with treatment condition occurred (assessment (completed, not-completed) $\times$ treatment (CBT, IPT); all $P>0.05)$.

\section{Primary outcomes}

Long-term recovery rates were $52.0 \%$ for the CBT group and $76.7 \%$ for the IPT group (Table 2, Fig. 2). The GEE logistic regression analysis showed a significant treatment $\times$ time effect on abstinence from binge eating $(P<0.001)$. Post hoc comparisons by time point did not reveal any significant between-treatment differences (all $P>0.01$ ). However, for CBT, a significant decline in recovery rates from post-treatment and 1-year follow-up to long-term follow-up was observed (both $P \geqslant 0.002$ ), whereas for IPT, abstinence rates did not change over the follow-up period (both $P>0.01$ ).

Long-term rates of remission to subclinical binge eating were $72.0 \%$ for the CBT group and $83.9 \%$ for the IPT group. The GEE logistic regression analysis of treatment $\times$ time did not show any significant effects on remission (all $P>0.05$ ). Long-term rates of improvement to a comparative level of eating disorder attitudes and behaviours (i.e. global EDE-Q score $\leqslant 2.47$ ) were $54.5 \%$ for the CBT group and $61.5 \%$ for the IPT group. The GEE logistic regression analysis showed a significant time effect on

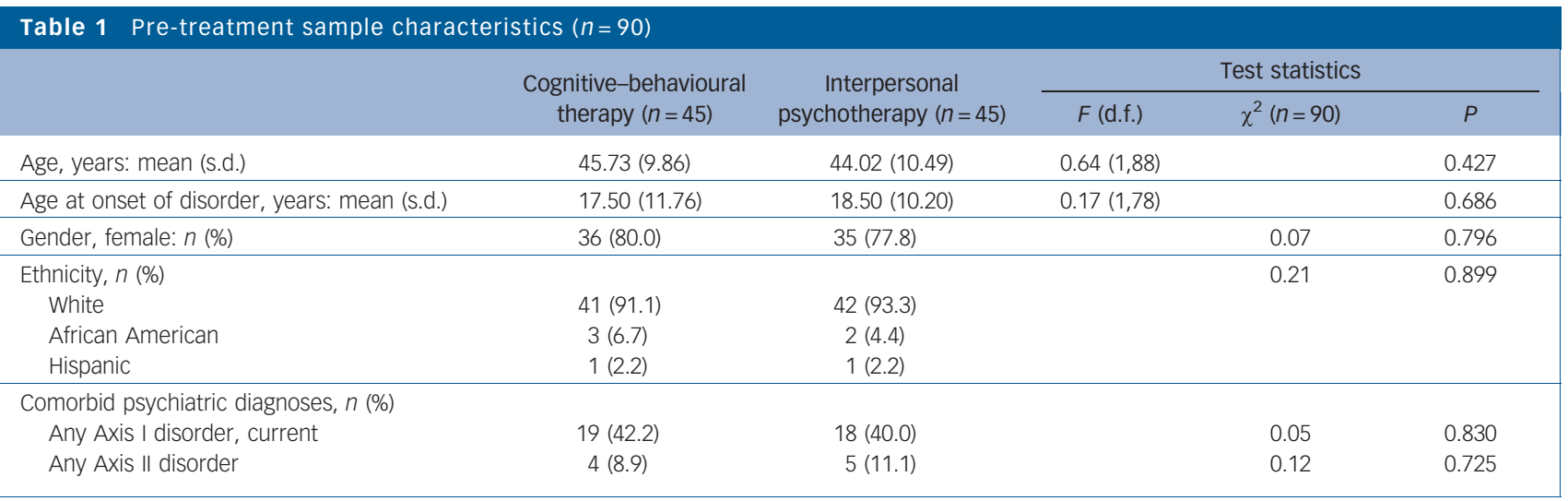


improvement $(P=0.049)$, but post hoc analyses did not reveal any change over the follow-up period (all $P>0.01$ ).

For all primary outcome variables, the sensitivity analysis revealed the same results with no replacement of missing data and with last observation carried forward. Multiple imputation results were largely consistent, with two exceptions: there was a significant time effect for remission and a significant treatment effect for clinically significant improvement (both $P<0.05$ ). Between-treatment effect sizes at long-term follow-up were small (recovery, $r=0.26$; remission, $r=0.14$; improvement, $r=0.07$ ).

Descriptive completer analyses revealed that 12 (27.3\%) CBT patients and $10(22.2 \%)$ IPT patients showed persistent recovery across all three follow-up assessments. From 1-year follow-up to long-term follow-up: $13(52.0 \%)$ patients in the CBT and $13(43.3 \%)$ in the IPT group maintained abstinence; no one $(0.0 \%)$ in the CBT and 10 patients $(33.3 \%)$ in the IPT group achieved abstinence; $6(24.0 \%)$ patients in the CBT and 3 $(10.0 \%)$ in the IPT group relapsed; and $6(24.0 \%)$ patients in the CBT and $4(13.3 \%)$ in the IPT group remained non-abstinent from objective bulimic episodes.

At long-term follow-up, $3(12.0 \%)$ individuals in the CBT group and $3(9.4 \%)$ in the IPT group were diagnosed with binge eating disorder. None of the participants were diagnosed with anorexia nervosa or bulimia nervosa or eating disorder not otherwise specified, and none revealed purging behaviour, fasting or intense exercising.

\section{Secondary outcomes}

The HLM analyses of treatment $\times$ time revealed significant time effects for all secondary outcome variables (all $P<0.001$ ), except BMI $(P=0.433$; online Table DS1). Post hoc analyses showed significant improvements in the number of days with objective bulimic episodes and EDE shape/weight concern, all EDE-Q indicators (except restraint) and depression at post-treatment, 1-year follow-up and long-term follow-up when compared with pre-treatment (all $P<0.01$ ). In contrast, the significant improvements of EDE-Q restraint and BSI anxiety present at post-treatment and 1-year follow-up when compared with pretreatment (both $P<0.01$ ) were no longer present at long-term follow-up $(P>0.01)$. Concerning the course over the follow-up period, the number of days with objective bulimic episodes and anxiety increased from post-treatment or 1-year follow-up to long-term follow-up (both $P<0.01$ ), whereas the reduced levels of EDE shape/weight concern, all EDE-Q indicators and depression were maintained from post-treatment or 1-year follow-up to long-term follow-up (all $P>0.01$ ).

The course of EDE-Q eating concern, shape concern and the global eating disorder psychopathology, and of EDE shape/weight concern showed significant interactions with treatment (all $P \leqslant 0.03)$ : for the CBT group, EDE-Q eating concern and EDE shape/weight concern worsened from 1-year follow-up to longterm follow-up (all post hoc $P<0.01$ ), whereas for IPT, there was an improvement in EDE-Q eating concern, shape concern and global eating disorder psychopathology from post-treatment to long-term follow-up $(P<0.01)$.

The sensitivity analysis revealed largely consistent results with no replacement of missing data, last observations carried forward and multiple imputation. The only difference was that for EDE-Q shape concern, the interaction effect was no longer significant with multiple imputation $(P<0.05)$. Most effect sizes between pre-treatment and long-term follow-up for eating disorder psychopathology were large $(0.97 \leqslant d \leqslant 2.10)$. Depression yielded a medium effect size $(d=0.53)$, and EDE-Q restraint, anxiety and BMI yielded small effect sizes $(d \leqslant 0.37)$. Between-treatment

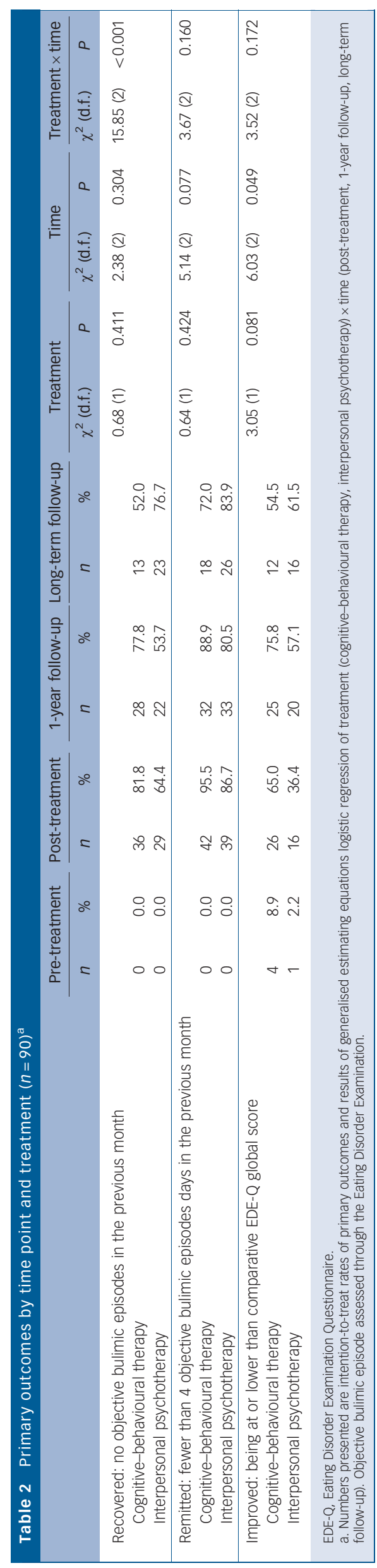




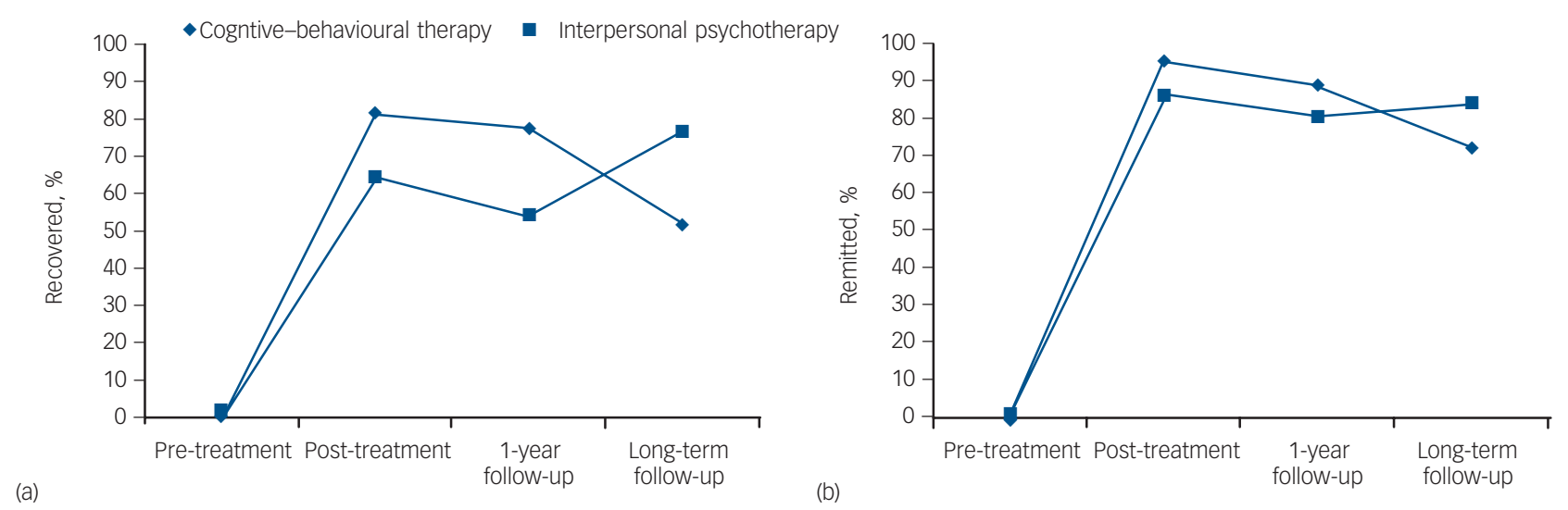

Fig. 2 Intention-to -treat recovery and remission rates.

(a) Intention-to-treat recovery rates, i.e. percentage of participants who had no objective bulimic episode in the previous month; (b) intention-to-treat remission rates, i.e. percentage of participants who had fewer than 4 days with objective bulimic episodes in the previous month.

effect sizes for all secondary outcomes at long-term follow-up were small $(0.18 \leqslant d \leqslant 0.49)$.

Descriptive completer analyses on healthcare utilisation showed that between 1-year follow-up and long-term follow-up, $20(80.0 \%)$ patients in the CBT group and $27(84.4 \%)$ in the IPT group had received treatment for eating or weight problems (psychotherapy: CBT group 11 (44.0\%), IPT group 12 (37.5\%); pharmacotherapy: CBT group $13(52.0 \%)$, IPT group 15 (46.9\%); consultation, for example with a dietician: CBT group 8 (32.0\%), IPT group 16 (50.0\%); alternative treatment, for example hypnosis: CBT group 7 (28.0\%), IPT group $9(28.1 \%))$. Healthcare utilisation was slightly associated with treatment condition (all $|r| \leqslant 0.18)$.

\section{Discussion}

\section{Main findings}

The current long-term follow-up study documented a substantial and long-lasting efficacy of both CBT and IPT for binge eating disorder, with full recovery from binge eating in $64.4 \%$ of patients. These data are consistent with 2-year follow-up recovery rates found in other clinical trials for binge eating disorder. ${ }^{10,11}$ Both CBT and IPT yielded comparable long-term rates of remission to a subclinical level of binge eating in $80.0 \%$ of patients and of clinically significant improvement of the associated eating disorder psychopathology in $58.0 \%$. The persistence of improvements was also reflected in low rates of binge eating disorder and in absence of compensatory behaviours or of any other eating disorder, as determined by the abbreviated EDE. In addition, most secondary outcomes of the associated eating disorder and general psychopathology showed significant and large improvements when compared with pre-treatment levels, although single outcomes, including objective bulimic episode days (the key symptom of binge eating disorder), restraint, and anxiety, showed tendencies toward relapse. Despite the overall favourable long-term outcome and in light of high rates of additional treatment-seeking following CBT and IPT, careful monitoring of these symptoms appears essential for early identification of the subset of patients showing reoccurrence of eating disorder symptoms over time.

There was some indication that CBT and IPT involved a differential time course over the follow-up period, although treatments did not differ in recovery rates at any time point. Abstinence from binge eating was stable over the follow-up period in the IPT group, whereas there was a significant tendency to relapse among patients in the CBT group. Concomitantly, reduction of eating disorder psychopathology in the IPT group was better maintained or further improved over the follow-up period, whereas for the CBT group psychopathology worsened from 1-year follow-up to long-term follow-up. This differential time course is similar to the 'catching up' effect of IPT that has previously been found in bulimia nervosa treatment. ${ }^{20,21}$ In two studies, IPT emerged as inferior to CBT at the end of treatment, but IPT patients showed continued improvement at 1-year follow-up, levelling off between-treatment differences. Based on the underlying theories of CBT and IPT, one might speculate that the focus on improving interpersonal relationships prepares individuals more comprehensively for the social challenges of daily life than the more rapidly acting, more eating disorderfocused CBT treatment, which reaches its efficacy earlier. Further research is warranted to clarify mechanisms of action of CBT versus IPT for binge eating disorder.

\section{Body mass index}

Body mass index was stable throughout the follow-up period, suggesting that the course of weight gain that is characteristic of treatment-seeking individuals with binge eating disorder ${ }^{22}$ has sustainably been interrupted. Given a tendency among adults to gain $400 \mathrm{~g}$ of weight per year, ${ }^{23}$ a stabilisation of body weight as documented in the current study may have led to a small amount of decreased weight gain of approximately $1600 \mathrm{~g}$ over the assessment period. Stabilisation of body weight as documented in the current study is a positive finding, in and of itself, as it is one of the priorities of obesity prevention. ${ }^{23}$

\section{Methodological considerations}

Strengths of this study include the conduct of an additional longterm follow-up addressing a fairly large subsample of patients (55.6\%) of a well-controlled clinical trial on binge eating disorder. Although the long-term assessment was not pre-planned and was unfunded, the overall participation rate of $64.4 \%$ was adequate for this study's sample (corresponding to $35.8 \%$ of the initial study sample that, however, was not considered for participation in this study).

Regarding generalisability of findings, several aspects underscore the certainty of results. No measurable biases existed in sample selection and assessment completion regarding sociodemographic characteristics, treatment drop-out, adherence 
and any outcome from pre-treatment to 1-year follow-up. In order to account for missing data, intention-to-treat analyses allowing data from assessment non-completers to remain in the analyses were conducted. The results from these intention-to-treat analyses showed the same pattern of results when compared with different treatment of missing data. ${ }^{18}$ The current completer sample size generated sufficient power to detect a medium-tolarge effect size of treatment difference at long-term follow-up; effect sizes were reported in order to reflect smaller size differences. Of the patients who were or could have been consented for this study, $75.3 \%$ completed long-term assessment. Likely related to the longer time interval, assessment completion rates were gradually lower from post-treatment to 1-year follow-up to long-term follow-up.

A further limitation to this study is that although we collected data on interim treatments, their effects could not be separated out from long-term effects of CBT and IPT themselves. Other intervening factors (for example life events) were not controlled for. Finally, the study sample was mostly female and White, which limits generalisation of results to male and other ethnic groups.

\section{Implications}

This report on the long-term efficacy of two major out-patient treatments for binge eating disorder suggests that IPT is a viable treatment alternative to standard CBT. Both treatments yielded high rates of treatment response and long-term maintenance of therapeutic gains. To bolster this study's findings, replication of long-term efficacy in a larger sample and over a longer time period is warranted. Alternative evidence-based treatment options, such as more individualised or comprehensive treatment, or extended or additional treatment, ${ }^{24}$ should be considered for individuals with poor initial treatment response to further optimise long-term treatment effects of CBT and IPT.

\footnotetext{
Anja Hilbert, PhD, Integrated Research and Treatment Center, Adiposity Diseases, Department of Medical Psychology and Medical Sociology, University of Leipzig Medical Center, Leipzig, Germany; Monica E. Bishop, MD, Department of Psychiatry, Washington University School of Medicine, St. Louis, Missouri, USA; Richard I. Stein PhD, Department of Internal Medicine, Washington University School of Medicine, St Louis, Missouri, USA; Marian Tanofsky-Kraff, PhD, Department of Medical and Clinical Psychology, Uniformed Services University of the Health Sciences, Bethesda, Maryland, USA; Anne K. Swenson, PhD, University of Washington Counseling Center, Seattle, Washington, USA; R. Robinson Welch, PhD, Denise E. Wilfley, PhD, Department of Psychiatry, Washington University School of Medicine, St. Louis, Missouri, USA

Correspondence: Anja Hilbert, Integrated Research and Treatment Center, Adiposity Diseases, Behavioral Medicine, University of Leipzig, Stephanstrasse 9C, 04103 Leipzig, Germany. Email: Anja.Hilbert@medizin.uni-leipzig.de

First received 18 Nov 2010, final revision 7 Jul 2011, accepted 28 Jul 2011
}

\section{Funding}

This research was supported by grants R29MH51384, R29MH138403 and K24MH070446 from the National Institutes of Health $(\mathrm{NIH})$. A.H. was supported by grant $01 \mathrm{EO} 1001$ from the German Federal Ministry of Education and Research; R.I.S. was partly supported by a KL2 Career Development Award and the Clinical and Translational Science Award at Washington University School of Medicine (NIH grants KL2RR024994 and UL1RR024992).

\section{References}

1 American Psychiatric Association. Diagnostic and Statistical Manual of Mental Disorders (4th edn, revised) (DSM-IV-TR). APA, 2000.
2 Striegel-Moore RH, Franko DL. Should binge eating disorder be included in the DSM-V? A critical review of the state of evidence. Annu Rev Clin Psychol 2008; 4: 305-24.

3 Wonderlich SA, Gordon KH, Mitchell JE, Crosby RD, Engel SG. The validity and clinical utility of binge eating disorder. Int J Eat Disord 2009; 42: 687-75.

4 Brownley KA, Berkman ND, Sedway JA, Lohr KN, Bulik CM. Binge eating disorder treatment: a systematic review of randomized controlled trials. Int J Eat Disord 2007; 40: 337-48.

5 Wilson GT, Grilo CM, Vitousek KM. Psychological treatment of eating disorders. Am Psychol 2007; 62: 199-216.

6 Hay P, Bacaltchuk J, Stefano S, Kashyap P. Psychological treatments for bulimia nervosa and binging. Cochrane Database Syst Rev 2009; 4: CD000562.

7 Vocks S, Tuschen-Caffier B, Pietrowsky R, Rustenbach SJ, Kersting A, Herpertz S. Meta-analysis of the effectiveness of psychological and pharmacological treatments for binge eating disorder. Int J Eat Disord 2009; 43: $205-17$.

8 National Institute for Health and Clinical Excellence. Eating Disorders - Core Interventions in the Treatment and Management of Anorexia Nervosa, Bulimia Nervosa, and Related Eating Disorders. National Clinical Practice Guideline Number CG9. The British Psychological Society and Gaskell, 2004.

9 Wilfley DE, Welch RR, Stein RI, Spurrell, EB, Cohen LR, Saelens BE, et al. $A$ randomized comparison of group cognitive-behavioral therapy and group interpersonal psychotherapy for the treatment of overweight individuals with binge eating disorder. Arch Gen Psychiatry 2002; 59: 713-21.

10 Wilson GT, Wilfley DE, Agras WS, Bryson SW. Psychological treatments of binge eating disorder. Arch Gen Psychiatry 2010; 67: 94-101.

11 Devlin MJ, Goldfein JA, Petkova E, Liu L, Walsh BT. Cognitive behavioral therapy and fluoxetine for binge eating disorder: two-year follow-up. Obesity 2007; 15: 1702-9.

12 Fairburn CG, Cooper ZH. The Eating Disorder Examination. In Binge Eating. Nature, Assessment and Treatment (eds Fairburn CG, Wilson GT). Guilford Press, 1993.

13 Fairburn CG, Beglin SJ. The assessment of eating disorders: interview or selfreport questionnaire? Int J Eat Disord 1994; 16: 363-70.

14 Sysko R, Walsh BT, Fairburn CG. Eating Disorder Examination - Questionnaire as a measure of change in patients with bulimia nervosa. Int J Eat Disord 2005; 37: 100-6.

15 Derogatis LR. Brief Symptom Inventory. Clinical Psychometric Research, 1991.

16 Derogatis LR, Melisaratos N. The Brief Symptom Inventory: an introductory report. Psychol Med 1983; 13: 595-605.

17 Castellini G, Lapi F, Ravaldi C, Vannacci A, Rotella CM, Faravelli C, et al Eating disorder psychopathology does not predict the overweight severity in subjects seeking weight loss treatment. Compr Psychiatry 2008; 49: 359-63.

18 Sterne J, White I, Carlin J, Spratt M, Royston P, Kenward MG, et al. Multiple imputation for missing data in epidemiological and clinical research: potential and pitfalls. BMJ 2009; 338: b2393.

19 Cohen L. Statistics for Social Scientists. Sage Publications, 1990.

20 Agras WS, Crow SJ, Halmi KA, Mitchell JE, Wilson GT, Kraemer HC. Outcome predictors for the cognitive behavior treatment of bulimia nervosa: data from a multisite study. Am J Psychiatry 2000; 157: 1302-8.

21 Fairburn CG, Jones R, Peveler R, Carr SJ. Three psychological treatments for bulimia nervosa: A comparative trial. Arch Gen Psychiatry 1991; 48: 463-9.

22 Barnes RD, Blomquist KK, Grilo CM. Exploring pretreatment weight trajectories in obese patients with binge eating disorder. Compr Psychiatry 2011; 52: 312-8.

23 Rosell M, Appleby $P$, Spencer $E$, Key $T$. Weight gain over 5 years in 21,966 meat-eating, fish-eating, vegetarian, and vegan men and women in EPICOxford. Int J Obes 2006; 30: 1389-96.

24 Mitchell JE, Agras S, Crow S, Halmi K, Fairburn CG, Bryson S, et al. Stepped care and cognitive-behavioural therapy for bulimia nervosa: randomised trial. Br J Psychiatry 2011; 198: 391-7. 